\title{
Optic Neuritis: Hidden Manifestations and Mimickers
}

\author{
Adel Ekladious ${ }^{1,2 *}$ \\ ${ }^{1}$ Faculty of Health and Medical Sciences, University of Western Australia, Crawley, Australia \\ ${ }^{2}$ Royal Hobart Hospital, Hobart, Australia \\ Email: ekladiou@gmail.com
}

How to cite this paper: Ekladious, A. (2022) Optic Neuritis: Hidden Manifestations and Mimickers. Open Journal of Ophthalmology, 12, 1-15.

https://doi.org/10.4236/ojoph.2022.121001

Received: October 25, 2021

Accepted: December 28, 2021

Published: December 31, 2021

Copyright $\odot 2022$ by author(s) and Scientific Research Publishing Inc. This work is licensed under the Creative Commons Attribution International License (CC BY 4.0).

http://creativecommons.org/licenses/by/4.0/

\begin{abstract}
Optic neuritis is the most common cause of unilateral visual loss associated with orbital pain on ocular movements and impaired colour vision. It can present with anterior optic neuritis, papillitis, a swollen optic disc, retrobulbar neuritis with a normal optic disc, neuritis with oedema of the optic disc and the macular star or anterior ischemic neuropathy. It may be the only manifestation of a disease such as demyelination or associated with systemic diseases such as systemic lupus, giant cell arteritis, Sjogren's syndrome, herpes simplex, herpes zoster, Lyme disease, CMV, EBV, HIV, toxoplasmosis, West Nile virus, Chikungunya, dengue fever, Rift Valley fever, mumps, rubella, measles, cat scratch disease, tuberculosis, syphilis, rickettsia illnesses, $\mathrm{Q}$ fever, Whipple disease, brucellosis, leptospirosis, leprosy, toxocariasis, malaria, Cryptococcus, candidiasis, histoplasmosis, Aspergillosis, mucormycosis, Bacillus Chalmette-Guerin (BCG) vaccination, ethanol and methanol toxicity, sarcoidosis, neuromyelitis optica and Liber's hereditary optic neuropathy. A systemic approach to the diagnosis is of paramount importance in confirming, diagnosing and treating optic neuritis and underlying systemic diseases to provide definitive cures. Failure to diagnose optic neuritis and treat the cause may result in optic atrophy and permanent blindness. In this paper, we reviewed the diagnosis and differential diagnoses of optic neuritis, including arteritis, ischemic, neuroretina, and vasculitic causes.
\end{abstract}

\section{Keywords}

Optic Nerve, Myelitis, Neuromyelitis Optica, Papilledema, Devices Disease

\section{Approach to the Diagnosis and Differential Diagnosis of Optic Neuritis and Discussion}

Optic nerve examination can lead to the diagnosis of many systemic diseases and

${ }^{*}$ Address: ${ }^{1} 35$ Stirling Hwy, Crawley, Western Australia; ${ }^{2} 48$ Liverpool Street, Hobart, Tasmania, Australia; Associate Professor; Mobile: 61499449905. 
should be part of a routine examination even when there are no eye symptoms. It might gives rise to the diagnoses of life-threatening diseases like malignant hypertension and increases intracranial pressure due to brain tumors [1].

Examination of the optic nerve encompasses visual acuity, the field of vision, light and accommodation reflex, and fundoscopy [2] .

The components of the optic nerve include the intraocular portion (approximately 2 centimeters), the intraorbital portion (20 - 30 millimeters), the intracanalicular portion $(10 \mathrm{~mm})$ where the nerve runs through the optic canal which is firmly attached to the dura and subsequently susceptible to trauma, and the intracranial portion (12-15 mm) which extends to optic chiasma where both optic nerves join together [3].

The detailed examination of the optic nerve can exclude congenital anomalies such as drusen, optic atrophy secondary to retinal disease, inflammation or Leber's disease, ischemic non-atretic optic neuronitis, compressive disease, infiltrative optic neuritis due to malignancy, optic nerve meningioma and glioma, papilledema, ischemic optic neuropathy, Neuromyelitis Optic Spectrum Disorder (NMOSD), Anti-Myelin Oligodendrocyte Glycoprotein (MOG) optic neuronitis, infective optic neuronitis which (including Cat-Scratch disease, Bartonella henselae, syphilis, toxoplasmosis, toxocariasis, Rocky Mountain Spotted fever, herpes simplex, herpes zoster, Epstein Barr virus, Cytomegalovirus, Tularemia and Lyme disease (common in the United States) [4].

Examination of the fundus is easy and most of the medical students are trained on how to perform this examination. However, once these students start working, they often lose the skill as they carry stethoscope rather than ophthalmoscope unless they become a neurology or ophthalmology registrar. The swollen optic nerve needs to be taken very seriously. In this article, we discuss the significance of a swollen optic disc in one or both two eyes, and also whether a swollen disc reduces visual acuity or not [5].

\section{Swollen Optic Disc with Normal Visual Acuity}

\subsection{Drusen}

Drusen is the most common cause of unilateral optic nerve swelling with normal vision [6]. There is a familial tendency for drusen to occur in first-degree relative [6]. It occurs in $2.5 \%$ of the population [6]. Drusen is easily diagnosed if it has been recognized before.

Drusen, also known as pseudo papilledema, is located at the surface of the optic nerve and appears as yellow bumpy globules at the optic nerve head. Drusen can be confirmed by imaging such as optical coherence tomography (OCT) and optic nerve head autofluorescence [7]. Patients with Drusen do not have any peripapillary exudate or hemorrhage [7]. Imaging of the brain and orbit is important to exclude early optic nerve sheath meningioma which can be asymptomatic for some time [8]. Optic sheath meningioma will enhance in Magnetic Resonance with contrast. 
If the diagnosis is confirmed, the patient should have close follow-up with a neuro-ophthalmologist, who will decide on the time for stereotactic radiotherapy [8] [9].

\subsection{Non-Arteritic Anterior Ischemic Optic Neuropathy}

Early non-arteritic anterior ischemic optic neuropathy (see Figure 1) can be manifested by a swollen optic disc with normal vision. Usually there is no relative afferent pupillary defect. Occasionally there might be an enlarged blind spot. These patients should be monitored by an ophthalmologist as $40 \%$ of patients will develop symptomatic non-arteritic ischemic optic neuropathy [1]. The examination of the other eye may show a small cup-to-disc ratio which is a red flag that the patient will develop non-arteritic anterior ischemic optic neuropathy in that eye [10]. The patient with Type 1 Diabetes Mellitus can rarely develop papillitis with normal vision and a swollen optic disc [11] (see Figure 2).

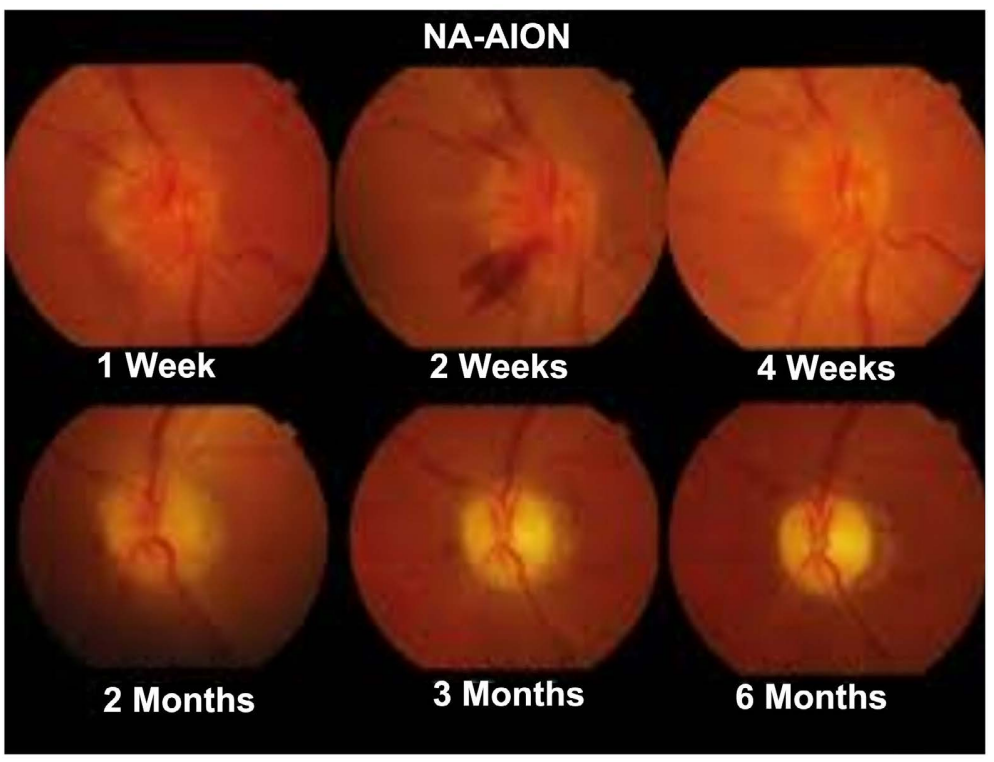

Figure 1. Non-arteritic anterior ischaemic optic neuritis.

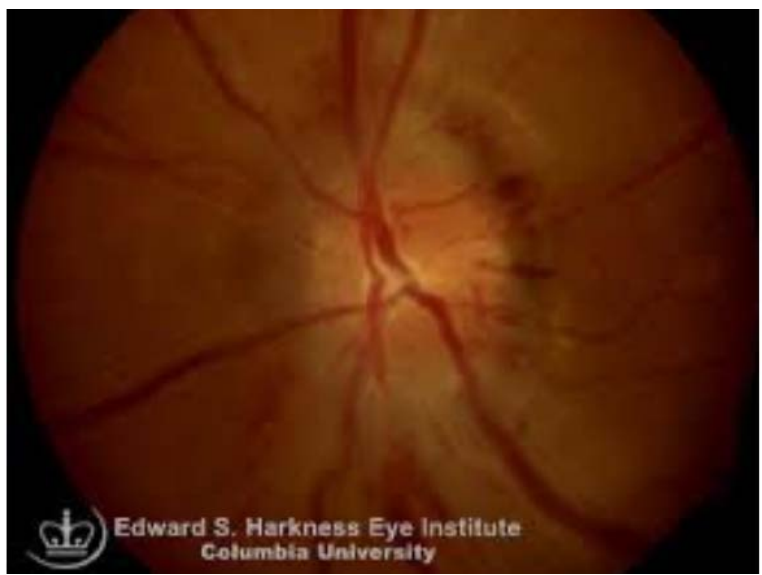

Figure 2. Diabetic papillopathy. 


\subsection{Non-Ischaemic Central Vein Occlusion}

Non-ischemic central vein occlusion can present with a swollen disc and normal vision. Fundus examination occasionally shows peripapillary hemorrhage. Few patients develop neovascular glaucoma. These patients should have fluorescein angiography to rule out ischemic optic neuropathy as the prognosis and the treatment is quite different [12].

\subsection{Optic Nerve Neuritis vs Optic Nerve Peri-Neuritis}

Orbital imaging can differentiate between optic nerve neuritis and optic nerve peri-neuritis where the optic nerve sheath, rather than the optic nerve, is swollen. The differential diagnosis is inflammatory granulomatous diseases such as sarcoidosis, granulomatosis with polyangiitis and Bechet's disease. Infective causes must also be ruled out (see Figure 3). It is rare for the disease to affect only one eye and cause unilateral papilledema (see Figure 4). Another sign to confirm papilledema is an absent retinal pulsation and enlargement of the blind spot. Vision is commonly normal except if the disease is undetected for a long period of time, and the patient develops optic atrophy (see Figure 5 and Figure 6). If choroidal neovascularization develops, the patient should be examined with Fluorescein Angiogram to rule out macular degeneration [13] [14].

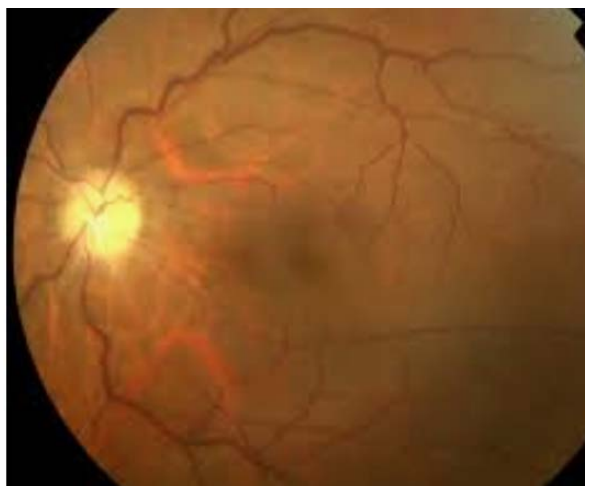

Figure 3. Infectious optic neuropathies.

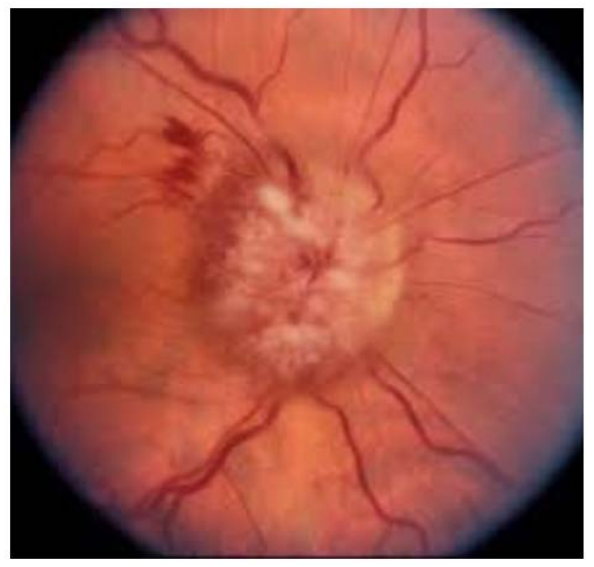

Figure 4. Papill oedema. 


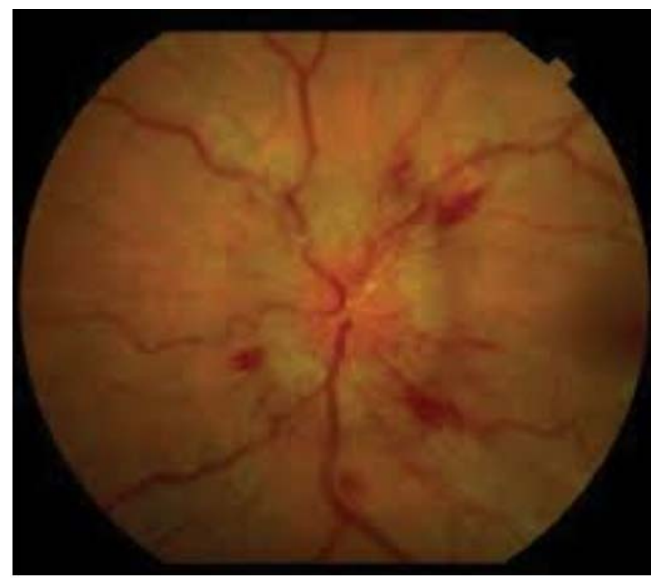

Figure 5. Optic atrophy due to long standing papilledema.

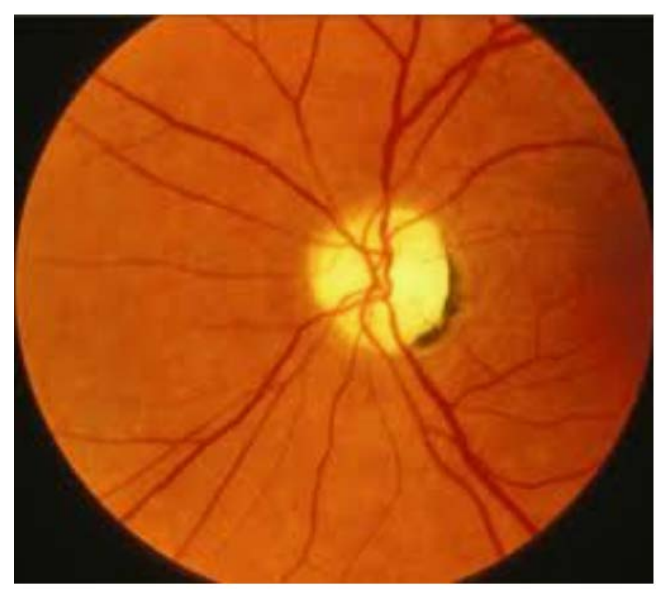

Figure 6. Optic atrophy due to untreated carotid AV fistula.

\subsection{Unilateral Optic Disc Swelling with Decreased Visual Acuity}

Optic neuritis is characterized by impaired vision, and usually affects young females. Pain with eye movement affects ten per cent of patients. These patients may have a peripapillary hemorrhage, and an orbital MR with contrast usually shows enhancement of the intraorbital optic nerve. The vision will improve spontaneously in $70 \%$ of patients. Patients will have a relative afferent papillary defect and a pale optic disc [15]. These patients should have an MRI of head and spine with gadolinium, OCT, visual evoked response testing, CSF testing for oligoclonal bands, and neurologist follow-up to exclude clinically isolated demyelinating disease [16].

\subsection{Non-Arteritic Ischaemic Optic Neuropathy}

Non-arteritic ischemic optic neuropathy is an unusual disease which affects older patients. Vision usually does not improve, and most patients have a peripapillary hemorrhage. Painful eye movements occur in few patients. If the optic nerve does not enhance with contrast, prognosis is guarded. Most patients have a small cup-to-disc ratio in the contralateral eye. Examination of visual field usually shows 
an altitudinal defect [17].

\subsection{Neuromyelitis Optica Spectrum Disorder}

Every non-Caucasian patient with optic neuritis, whether the myelitis is in one or two sides, or whether there is acute or subacute vision impairment, should be investigated for Neuromyelitis Optica Spectrum Disorder (NMOSD (Figure 7). NMOSD mainly affects women of child-bearing age within the posterior portion of optic nerve, with extension to the chiasma. The current clinical guideline recommends that positive serology of AQP4 IGG antibody is confirmed by cell-based serology, and one core clinical criteria which includes:

- Longitudinal extensive transverse myelitis: which spans over three or more contiguous vertebrae (usually cervical), with extension to the dorsal medulla, involving central grey matter of the cord causing anterior lateral cord syndrome;

- Acute postrema syndrome: characterized by nausea, vomiting, and intractable hiccups due to a peri-aqueductal lesion in mid brain and dorsal medulla;

- Narcolepsy and hypersomnolence: due to diencephalic, thalamic and hypothalamic involvement;

- Acute brainstem syndrome: causing facial palsy, ataxia, intractable itching, dizziness, and even death due to acute respiratory failure caused by involvement of respiratory centers. Early diagnosis is of paramount importance as patients respond to IV methylprednisolone and if required, plasmapheresis in severe disease. Once the disease is confirmed, patients need five years or more on immunosuppressive medication. Medications used to maintain remission include rituximab, azathioprine and mycophenolate mofetil [16] [17] [18] [19] [20].

\subsection{Myelin Oligodendrocyte Glycoprotein (MOG) Optic Neuritis}

MOG optic neuritis (Figure 8) usually affects males and females equally. It can

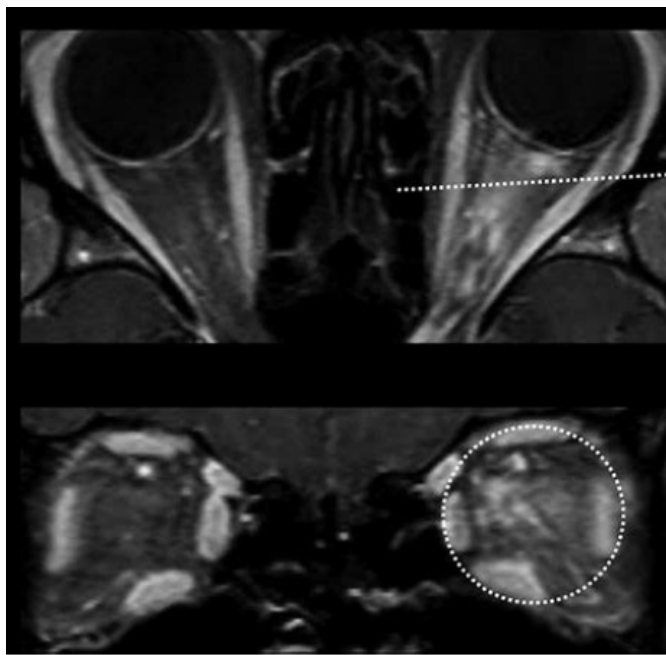

Figure 7. Optic neuritis due to AQP4 NMOSD. 

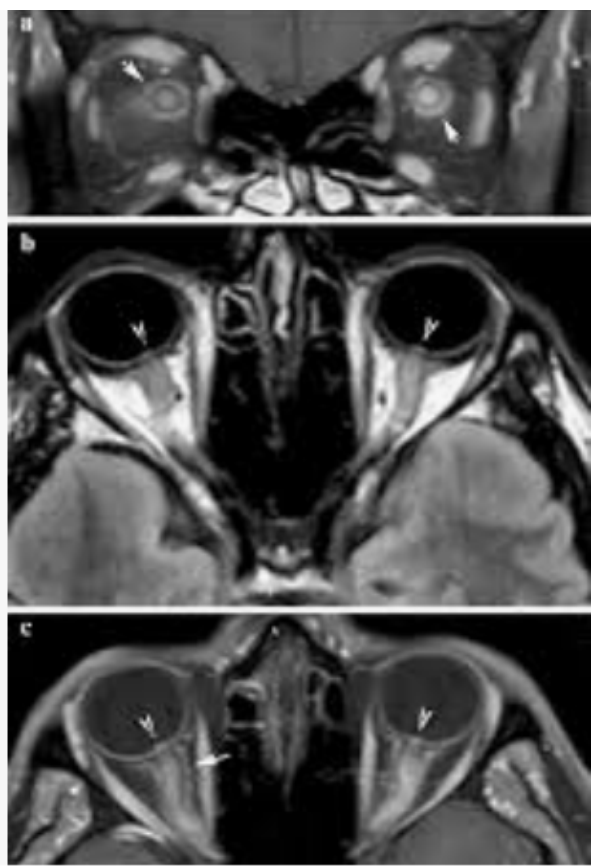

Figure 8. Patients with anti-MOG antibody.

present with simultaneous bilateral optic neuritis which involves the anterior part of optic nerve. MRI with contrast commonly shows perineural enhancement which is usually monophasic in phenotype. In contrast, NMOSD usually has a relapsing-remitting course, and is less likely to be associated with immunological disease and affect the lower part of the spinal cord and the conus. The spectrum of the disease includes acute disseminated encephalopathy disease confirmed by serological positivity for MOG [19]. CSF shows high protein with pleocytosisparticularly monocytes, lymphocytes or neutrophils.

Other differential diagnoses to consider and rule out include:

- Bechet's disease;

- Neuro-sarcoid granulomatous disease;

- Systemic lupus erythematosus;

- Ocular lymphoma;

- Primary angiitis of the CNS;

- Acute demyelinating encephalomyelitis;

- Susac syndrome;

- Infective ventriculitis;

- Dural spinal AV fistulae;

- Infarction of the anterior spinal nerve;

- Diffuse glioma;

- Antiphospholipid syndrome;

- Clinically acute isolated demyelination syndrome;

- Optica spinal multiple sclerosis;

- ANCA associated vasculitis;

- Large vessel vasculitis (giant cell arteritis). 
We will now discuss all differential diseases and highlight the main differentiating features for confirming or refuting the diagnosis of optic neuronitis.

\subsection{Neuro-Behcet Disease}

A chronic relapsing inflammatory disorder of idiopathic origin, mostly present in populations from Japan, Turkey and the Middle East. It is characterized by painful mouth, genital and eye ulceration, and a positive pathergy test. The two main types are parenchymatous and vascular. Neuro-Bechet Disease (see Figure 9) can cause progressive atrophy of the optic disc and severe loss of vision [17].

\subsection{Neuro-0phthalmic Sarcoid}

Sarcoid disease commonly affects the lungs. The manifestation of Neuro-Ophthalmic Sarcoid (see Figure 10) affects up to 7\% of sarcoidosis patients. The optic disc may be swollen and accompanied by exudate in the retina (sarcoid neuroretinitis) or develop an optic head granuloma. Other commonly affected cranial nerves include the oculomotor and the facial nerve. Patients may develop decreased visual acuity and diplopia. Patients with normal vision should be investigated by OCT to exclude retrobulbar neuritis, especially if patients have pain with eye movement. The other rare manifestation of ocular sarcoidosis is Horner

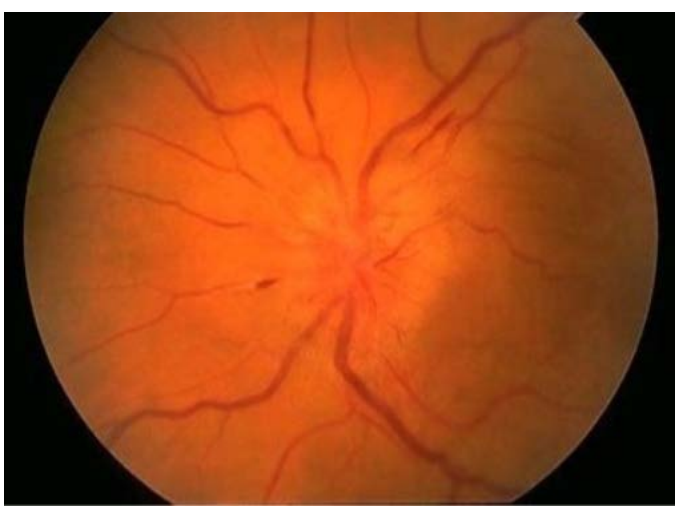

Figure 9. Optic neuritiis in Neuro-Behcets disease.

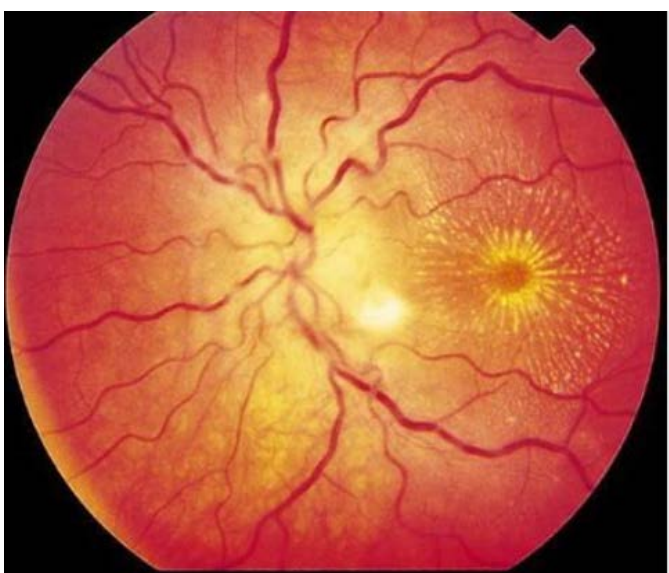

Figure 10. Optic neuritis due to sarcoid. 
syndrome. Other non-ocular signs in sarcoidosis are hypercalcemia due to elevated 1-25 hydroxy-vitamin D, erythema nodosum, Lupus Pernio, macular popular lesions, pleocytosis in the CSF, and in 10\%, low sugar within the CSF. A CT-PET scan may show an area which could be a target for biopsy to confirm non-caseating granuloma [19] [20].

\subsection{Ocular Lymphoma}

Ocular lymphoma is a very rare tumeur, which could be primay B cell lymphoma or secondary to systemic lymphoma, the most common manifistation is non granulomatous anerior uveitis and vitritis where the disease is often bilateral, occulr $\mathrm{T}$ cell lymphoma is very rare, Optical Cohornse Tomography (OCT) is important tool for investigation.

Ocular lymphoma will come to the attention after failure to respond to treatment, ocular lymphoma responds to steroid and causes delay in the diagnosis, if specific investigation was not performed before commencement of steroid, opthalmogist should have a low threshold to involve other subspecialty like hematologist and oncologist to be involved, disease should be managed by multidisciplinary team [21] [28].

Demyelinating optic neuritis may occur as a paraneoplastic manifestation of Hodgkin's lymphoma (see Figure 11). It is usually painless and manifested by a loss of vision. Treatment of the cancer usually leads to a cure of the optic neuritis [21]. Non-Hodgkin Lymphoma affects the optic nerve through direct involvement of the orbit, causing compression of the optic nerve. Occasionally primary infiltration of the nerve or sheath can cause optic neuritis [21] (Figure 12).

\subsection{Primary Angiitis of the CNS}

Primary angiitis of the CNS is a rare vasculitic disease. It has a multifaceted set of clinical signs and symptoms. Leptomeninges are commonly involved, and an MRI Brain is nonspecific. Both parenchymal and vascular lesions predominantly affect the anterior cerebral circulation. The lesions may be proximal, unilateral

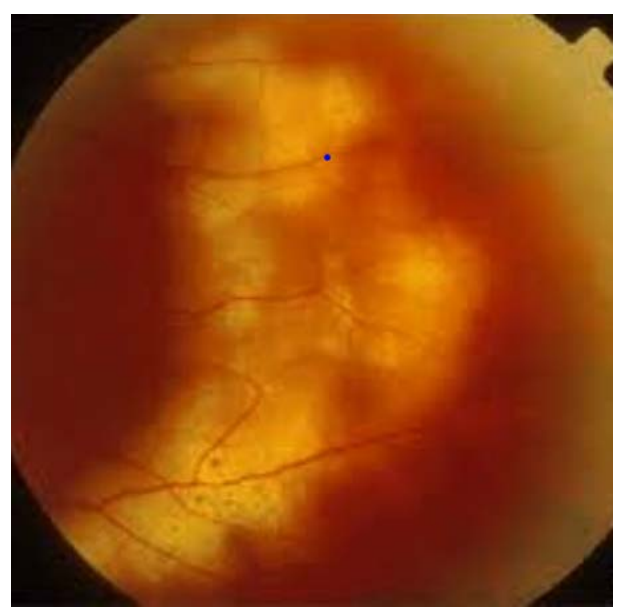

Figure 11. Intralocular lymphoma. 
and multifocal. There is a good complement between MRI and MRA in the anterior circulation, which usually shows stenotic occlusions, a beaded appearance, aneurysms, and eccentric vessel irregularities. Despite all imaging studies, brain biopsy is gold standard to confirm the diagnosis CNS Angiitis [22]. All other differential diagnoses must be ruled out before beginning treatment with steroids and cyclophosphamide [22].

\subsection{Acute Demyelinating Encephalomyelitis (ADEM)}

ADEM is a rare neurological disease affecting children and young adolescents. It is characterised by a polyfocal monosymptomatic course of disease. Most patients have a history of vaccination or viral illness within the preceding one or two weeks [23].

Initial symptoms include fever, headache, fatigue and malaise. Neurologic manifestations include optic neuritis, transverse myelitis, ataxia, cranial nerve palsy, elevated CSF protein, pleocytosis (mainly lymphocytes and monocytes) and oligoclonal band usually present transiently in up to $10 \%$ of patients [23]. Some studies reported that MOG is detected in the serum in up to $40 \%$ of the patients [24]. Treatment is IV methylprednisolone, 1 gram per day for 5 days. Full recovery is expected in $80 \%$ of patients. In patients who do not respond to steroids, plasma exchange, or where contraindicated, intravenous immunoglobulin are other options for treatment [24].

\subsection{Susac Syndrome}

Susac syndrome is a rare CD8 mediated autoimmune disease characterized by branch retinal artery occlusion, hearing loss, encephalopathy, focal neurologic defects and scotoma. It affects small vessels of the brain, retina and inner ear. Females are more commonly affected than males with a ratio of 5:1. Susac syndrome usually presents with a headache and systemic symptoms, and elevated protein within the CSF. Brain biopsy usually shows micro infarct of the arteries and loss of myelin, axons and neurons.

Important differential diagnoses include Multiple sclerosis, CN Vasculitis, limbic encephalitis, stroke, Cogan syndrome, and CADASIL syndrome (cerebral autosomal dominant arteriopathy with subcortical infarcts and leukoencephalopathy.

Fluoresceine Angiogram is a standard investigation to confirm branch retinal occlusion. OCT is noninvasive and can diagnose retinal vessel ischemia and optic neuritis. Empirical treatment is dependent on best practice but may include steroids, mycophenolate, rituximab, plasma exchange, tissue necrosis factor inhibitors and Natalizumab [25].

\subsection{Infective Ventriculitis}

Infective ventriculitis usually results from catheter-related coagulase negative staphylococcal infection. Common gram-negative organisms are E. coli, Klebsiella, 
Pseudomonas aeruginosa. The patient is usually visually unwell. CSF culture is important to guide early treatment [26].

\subsection{Spinal Dural A-V Fistulae}

A spinal dural arteriovenous fistula (Figure 13) is a rare acquired vascular disease. There are few cases of reported optic nerve neuritis amongst patients with this condition. It is often missed unless patient has associated transverse myelitis [26]. Patient may have added ocular signs such as diplopia, proptosis, epiphora and decreased vision acuity. Symptoms increase with effort and decrease at rest due to decreased brain perfusion due to decreased $\mathrm{A}-\mathrm{V}$ gradient due to the fistula [27] [28] [29] [30].

\subsection{ANCA Associated Vasculitis}

ANCA associated vasculitides that may cause optic neuritis include microscopic polyangiitis, granulomatosis with polyangiitis and Churg-Strauss vasculitis. The mechanism of optic neuropathy may be due to compression or vasculitis. It is likely to be associated with active urinary sediment due to necrotizing glomerulonephritis.

Few case reports have described a group of patients with sinus and orbital disease without glomerulonephritis. C-ANCA is positive in $90 \%$ of patient with granulomatosis with polyangiitis without glomerulonephritis. Urine sediment is

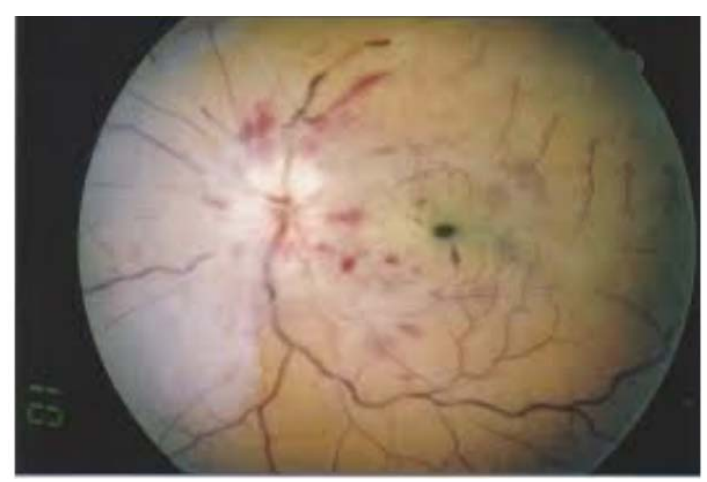

Figure 12. Systemic lymphomas.

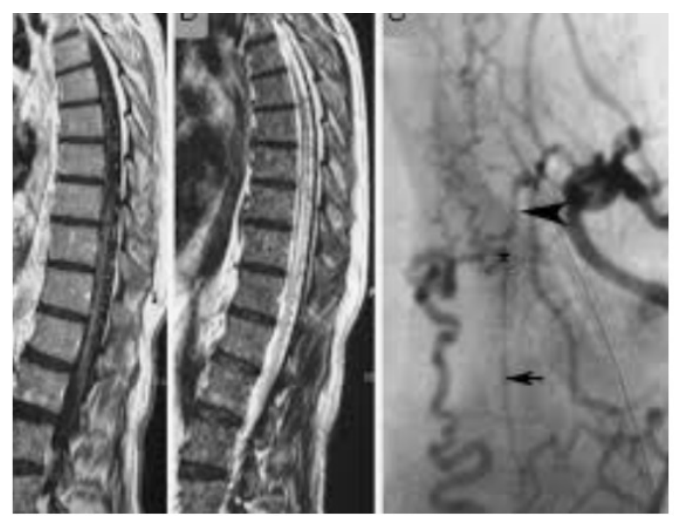

Figure 13. Spinal dural arteriovenous fistulas 


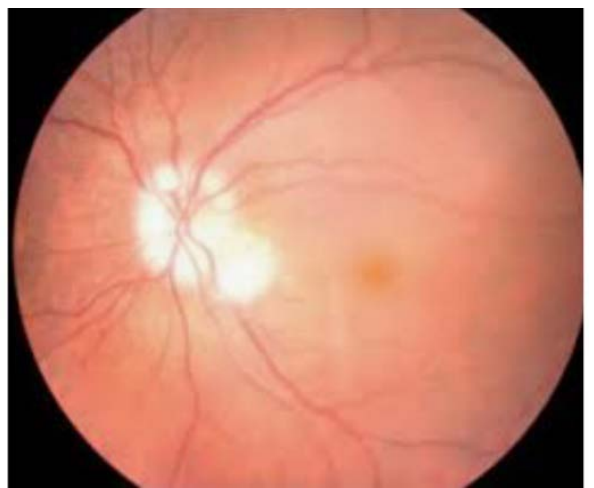

Figure 14. Giant cell arteritis.

bland and been diagnosed as ophthalmic granulomatosis [31] [32]. P-ANCA is positive in most patients with microscopic polyangiitis and Churg-Strauss disease.

Few studies reported an association between basement membrane disease like Alport syndrome and optic neuritis as both diseases affect collagen fiber type IV.

\subsection{Large Vessel Vasculitis (Giant Cell Arteritis)}

Anterior arteritic ischemic optic neuritis due to Giant Cell Arteritis (see Figure 14) is a serious disease and ophthalmic emergency as it can cause permanent blindness if immediate treatment is not started before waiting for confirmation of the disease [31].

Common systemic symptoms are headache, fatigue, tiredness, proximal muscle tenderness and weakness. Fundoscopy usually shows a pallid appearance and bilateral disc swelling with a chalky appearance. Elevated ESR and CRP are common in $85 \%$ of patients.

Bedside ultrasound is a valuable non-invasive tool for diagnosis. The "Halo sign", where the cut-off values for temporal vessel are $0.3-1.0$ and $1.0-2.0$ for axillary vessels, and inflammatory vessels are not compressible. Specificity is up to $90 \%$ in the experienced technician; however, ultrasound of the extracranial vessels is not diagnostic. Temporal artery biopsy is invasive and has a low sensitivity as Giant Cell Arteritis is a patchy disease, not necessarily affecting the entire vessel.

\section{Conclusions}

Optic neuritis is a serious disease should not be missed. It was initially thought to be benign if it responded well to steroids. Early recognition of optic neuritis is essential for prompt referral to an ophthalmologist. Patients with optic neuritis may need to be treated by a multidisciplinary team including an ophthalmologist, neurologist, infectious disease specialist, oncologist, geneticist and/or immunologist.

Visual MRI, visual evoked response, cerebrospinal studies and optical coherence tomography have become essential tools in the investigation, timely diag- 
nosis and treatment of optic neuritis.

\section{Acknowledgements}

Associate Professor Adel Ekladious thanks Cassandra Byers and Ramana Waran for editing the manuscript. Adel Ekladious acknowledged Associate Professor Philip Braslins for his advice.

\section{Conflicts of Interest}

The author declares no conflicts of interest regarding the publication of this paper.

\section{References}

[1] Bettman, J.W., Daroff, R.B., Sandres, M.D. and Joyt, W.F. (1968) Papilledema and Asymptomatic Intra Cranial Hypertension in SLE. Archives of Ophthalmology, 80, 180-193. https://doi.org/10.1001/archopht.1968.00980050191007

[2] Green, G.L., Lesse, L.L. and Loewnstein, J.L. (1980) Ischemic Optic Neuropathy in Chronic Papilledema. Archives of Ophthalmology, 98, 502-504. https://doi.org/10.1001/archopht.1980.01020030498013

[3] Karam, E.Z. and Hedges, T.R. (2005) Optical Coherence Tomography for Retinal Nerve Fiber Layer in Mild Papilledema and Pseudo Papilledema. British Journal of Ophthalmology, 89, 294-298. https://doi.org/10.1136/bjo.2004.049486

[4] Sarks, S.H., Arnold, J.J., Killingsworth, M.C. and Sarks, J.P. (1999) Early Drusen Formation in Normal and Aging Eye and Their Relation to Age Related Maculopathy: A Clinicopathological Study. British Journal of Ophthalmology, 83, 358-368. https://doi.org/10.1016/S0161-6420(02)01146-6

[5] Bressler, N.M., Munoz, B. and Maguire, M.G. (1995) Five Year Incidence and Disappearance of Drusen and Retinal Pigment Epithelial Abnormalities: Waterman Study. Archives of Ophthalmology, 113, 301-308. https://doi.org/10.1001/archopht.1995.01100030055022

[6] Kline, E. and Lanning, B. (2014-2015) Neuro-Ophthalmology: Basic and Clinical Science Series. American Academy of Ophthalmology, San Francisco.

[7] Dutton, J.J. (1992) Optic Nerve Sheath Meningiomas. Survey of Ophthalmology, 37, 167-183. https://doi.org/10.1016/0039-6257(92)90135-g

[8] Shapey, J., Sabin, H., Danesh Mayer, H.V. and Kaye, A.H. (2013) Diagnosis of the Optic Nerve Sheath: 1045-1056 Meningiomas. Journal of Clinical Neuroscience, 20, 1045-1056. https://doi.org/10.1016/j.jocn.2013.03.008

[9] Arnold, A.C. (2003) Pathogenesis of Nonatretic Anterior Ischemic Optic Neuropathy. Journal of Neuro-Ophthalmology, 23, 157-163. https://doi.org/10.1097/00041327-200306000-00012

[10] Boone, M.I., Massry, G.G., Frankel, R.A., Holds, J.B. and Chung, S.M. (1996) Visual Outcome in Bilateral Nonarthritic Anterior Ischemic Optic Neuropathy. Ophthalmology, 103, 1223-1228. https://doi.org/10.1016/s0161-6420(96)30519-8

[11] Slagle, W.S., Musick, A.N. and Eckermann, D.R. (2009) Diabetic Papillopathy and Its Relationship to Optic Nerve Ischemia. Optometry and Vision Science, 86, e395-e403. https://doi.org/10.1097/OPX.0b013e318198927c

[12] Jarius, S., Paul, F., Aktas, O., Asgari, N., Dale, R.C., de Seze, J., et al. (2018) MOG Encephalitis: International Recommendation on Diagnosis and Antibody Testing. 
Journal of Neuroinflammation, 15, Article No. 134. https://doi.org/10.1186/s12974-018-1144-2

[13] Narayan, R., Simpson, A., Fritsche, K., Salama, S., Pardo, S., Mealy, M., et al. (2018) MOG Antibody Disease: A Review of MOG Antibody Seropositive Neuromyelitis Optica Spectrum Disorder. Multiple Sclerosis and Related Disorders, 25, 66-72. https://doi.org/10.1016/j.msard.2018.07.025

[14] Wingerchuk, D.M., Lennon, V.A., Pittock, S.L., Lucchinetti, C.F. and Weinshenker, B.G. (2006) Revised Diagnostic Criteria for Neuromyelitis Optica. Neurology, 66, 1485 1489. https://doi.org/10.1212/01.wnl.0000216139.44259.74

[15] Wingerchuk, D.M., lennon, V.A., Lucchinetti, C.F., Pittock, S.J. and Weinshenker, B.G. (2007) The Spectrum of Neuromyelitis Optica. The Lancet Neurology, 6, 805-815. https://doi.org/10.1016/S1474-4422(07)70216-8

[16] Kansu, T., Kirkali, P., Kansu, E. and Zileli, T. (1989) Optic Neuropathy in Behçet's Disease. Journal of Neuro-Ophthalmology, 9, 277-289

[17] Koczman, J.J., Rouleau, J., Gaunt, M., Kardon, R.H., Wall, M. and Lee, A.G. (2008) Neuro-Ophthalmic Sarcoidosis: The university of Iowa Experience. Seminars in Ophthalmology, 23, 157-168. https://doi.org/10.1080/08820530802007382

[18] Hunninghake, G.W., Costbel, U., Ando, M., et al. (1999) ATS/ERS/WASOG Statement on Sarcoidosis American Thoracic Society/World Association /World Association of Sarcoidosis and Other Granulomatous Disorders. Sarcoidosis, Vasculitis and Diffuse Lung Disease, 16, 149-173.

[19] Behbehani, R.S., Vacarezza, N., Sergott, R.C., Bilyk, J.R., Hochberg, F. and Savino, P.J. (2005) Isolated Optic Nerve Lymphoma Diagnosed by Optic Nerve Biopsy. American Journal of Ophthalmology, 139, 1128-1130. https://doi.org/10.1016/j.ajo.2004.12.006

[20] Vattoth, S., Compton, C.J., Roberson, G.H. and Vaphiades, M.S. (2013) Susac Syndrome. A Differential Diagnosis of Demyelination. Neurosciences (Riyadh), 18, 74-78.

[21] Siva, A. (2001) Vasculitis of the Nervous of the Nervous System. Journal of Neurology, 248, 451-468. https://doi.org/10.1007/s004150170154

[22] Tselis, A.C. and Lisak, R.P. (1998) Acute Disemminated Encephalomyelitis. In: Antel, J., Birnbaum, G. and Hartung, H.P., Eds., Clinical Neuroimmunology, Blackwell Science, Boston, 116-147.

[23] Biousse, V., Touboul, P.J., D’Anglejan-Chatillon, J., Lévy, C., Schaison, M. and Bousser, M.G. (1998) Ophthalmologic Manifestations of Internal Carotid Artery Dissection. American Journal of Ophthalmology, 126, 565-577. https://doi.org/10.1016/S0002-9394(98)00136-6

[24] Dutton, J.J. (1994) Gliomas of the Anterior Visual Pathway. Survey of Ophthalmology, 38, 427-452. https://doi.org/10.1016/0039-6257(94)90173-2

[25] Balcer, L.J. (2006) Optic Neuritis. New England Journal of Medicine, 354, 1273-1280. https://doi.org/10.1056/NEJMcp053247

[26] Behbehani, R., Sergott, R.C. and Savino, P.J. (2004) The Antiphospholipid Antibody Syndrome, Diagnostic Aspects. Current Opinion in Ophthalmology, 15, 483-485. https://doi.org/10.1097/01.icu.0000144389.97491.52

[27] Nakashima, I., Fukazawa, Y., Ota, K., Nohara, C., Warabi, Y., Ohashi, T., et al. (2007) Two Subtypes of Optica-Spinal Form of Multiple Sclerosis in Japan: Clinical and Laboratory Features. Journal of Neurology, 254, 488-492. https://doi.org/10.1007/s00415-006-0400-z

[28] Townsend, J.K., Dubovy, S.R., Pasol, J. and Lam, B.L. (2013) Transient Optic Neuritis as the Initial Presentation of Central Nervous System Involvement by Pre-B Cell 
Lymphocytic Leukemia. Journal of Neuro-Ophthalmology, 33, 162-164. https://doi.org/10.1097/WNO.0b013e318281b84d

[29] Hamada, J., Morioka, M., Kai, Y., Sakurama, T. and Kuratsu, J. (2006) Spontaneous Arteriovenous Fistula of the Orbit: Case Report. Surgical Neurology, 65, 55-57. https://doi.org/10.1016/j.surneu.2005.03.043

[30] Van den Berg, R., Smagge, L.E., Saeed, P. and Majoie, C.B. (2009) Dural Arteriovenous Fistula of the Optic Nerve Sheath. Orbit, 28, 417-419. https://doi.org/10.3109/01676830903104769

[31] Hayreh, S.S. and Zimmerman, B. (2003) Visual Deterioration in Giant Cell Arteritis Patients While on High Dose Corticosteroid Therapy. Ophthalmologica, 110, 239-259. https://doi.org/10.1159/000070631

[32] Ahmed, M., Niffenegger, J.H., Jakobiec, F.A., Ben-Arie-Weintrob, Y., Gion, N., Androudi, S., et al. (2008) Diagnosis of Limited Ophthalmic Wagner Granulomatosis; Distinive Pathological Features with ANCA Test Confirmation. International Ophthalmology, 28, 35-46. https://doi.org/10.1007/s10792-007-9109-y 